\title{
LIFTING PUZZLES IN DEGREE FOUR
}

\author{
CRIS POOR ${ }^{凶}$, NATHAN C. RYAN and DAVID S. YUEN
}

(Received 19 June 2008)

\begin{abstract}
We identify the majority of Siegel modular eigenforms in degree four and weights up to 16 as being Duke-Imamoğlu-Ikeda or Miyawaki-Ikeda lifts. We give two examples of eigenforms that are probably also lifts but of an undiscovered type.
\end{abstract}

2000 Mathematics subject classification: primary 11F46, 11F60; secondary 65D20, 65-04.

Keywords and phrases: Siegel modular forms, Satake parameters, Miyawaki-Ikeda lifts.

\section{Introduction}

For degree four, vector spaces of Siegel modular cusp forms are known through weights $k \leq 16$. For weight 16 there are seven eigenforms: three quadratic pairs and one rational cusp form. From the work of Ikeda, the $L$-functions of two of the quadratic pairs are known. In this article, we compute the Euler 2-factors for the remaining quadratic pair and for the rational eigenform. Some of the roots of these Euler factors are not unimodular and so, presumably, there are two new lifts to be discovered. If not, the generalized Ramanujan-Petersson conjecture needs significant modification. The unexplained Euler 2-factors do factor in interesting ways, see Table 2. In this article we survey all $L$-functions for degree four and weights $k \leq 16$. We both explain how to compute Euler factors in higher degree and actually compute some. We find congruences between particular Euler factors and prove the nonvanishing of particular Miyawaki lifts.

Lifting conjectures have been motivated by the computation of Euler factors of $L$-functions of Siegel modular Hecke eigenforms. Let $S_{n}^{k}$ denote the Siegel modular cusp forms of degree $n$ and weight $k$. For Hecke eigenforms in $S_{2}^{10}$ and $S_{2}^{12}$, Kurokawa [15] computed some Euler factors and noted their factorization into Euler products of elliptic modular cusp forms and zeta functions.

1. Saito-Kurokawa-Maass lift. For even weights $k$, each eigenform $f \in S_{1}^{2 k-2}$ corresponds to an eigenform $F \in S_{2}^{k}$ such that the standard $L$-function of $F$

(C) 2009 Australian Mathematical Society 0004-9727/2009 \$16.00 
factors

$$
L(F, s, \mathrm{st})=\zeta(s) L(f, s+k-1) L(f, s+k-2) .
$$

This was proven by Maass, Andrianov and Zagier, see [5]. For eigenforms in $S_{3}^{12}$ and $S_{3}^{14}$, Miyawaki [16] computed some Euler factors and used their factorizations to give two conjectures. We rephrase them as follows.

2. Miyawaki Conjecture I. For even $k$, each pair of eigenforms $f \in S_{1}^{2 k-4}$ and $g \in S_{1}^{k}$ corresponds to an eigenform $F \in S_{3}^{k}$ such that

$$
L(F, s, \mathrm{st})=L(f, s+k-2) L(f, s+k-3) L(g, s, \mathrm{st}) .
$$

3. Miyawaki Conjecture II. For even $k$, each pair of eigenforms $f \in S_{1}^{2 k-2}$ and $g \in S_{1}^{k-2}$ corresponds to an eigenform $F \in S_{3}^{k}$ such that

$$
L(F, s, \mathrm{st})=L(f, s+k-1) L(f, s+k-2) L(g, s, \mathrm{st}) .
$$

Duke and Imamoḡlu studied the factorization of the Euler factors of the Schottky form in $S_{4}^{8}$ and, in analogy with the Saito-Kurokawa lift, conjectured a lifting proven by Ikeda [9].

4. Duke-Imamoḡlu-Ikeda Lift. For even $k, n$ and $(n+k) / 2$, each eigenform $f \in$ $S_{1}^{k}$ corresponds to an eigenform $I_{n}(f) \in S_{n}^{(n+k) / 2}$ whose standard $L$-function is

$$
L\left(I_{n}(f), s, \mathrm{st}\right)=\zeta(s) \prod_{i=1}^{n} L\left(f, s+\frac{n+k}{2}-i\right) .
$$

Ikeda has also solved Miyawaki's first conjecture with an additional natural nonvanishing condition, see [8]. See also [12] for the resolution of a germane conjecture in [8]. In contrast, Miyawaki's second conjecture remains open. Given a complex function $g$ on the Siegel upper half space, define $g^{c}$ by $g^{c}(Z)=\overline{g(-\bar{Z})}$.

5. Miyawaki-Ikeda Lift. Let $k, n+r$ and $\hat{k}=(n+r+k) / 2$ be even integers with $r \leq n$. Each pair of eigenforms $f \in S_{1}^{k}$ and $g \in S_{r}^{\hat{k}}$ corresponds to an element $M_{n}(f, g) \in S_{n}^{\hat{k}}$ via $M_{n}(f, g)\left(Z_{n}\right)=\left\langle I_{n+r}(f)\left(Z_{n} \oplus Z_{r}\right), g^{c}\left(Z_{r}\right)\right\rangle$, where $\langle\cdot, \cdot\rangle$ is the Petersson inner product. If $M_{n}(f, g)$ is nontrivial, then it is an eigenform for the even Hecke algebra and

$$
L\left(M_{n}(f, g), s, \mathrm{st}\right)=L(g, s, \mathrm{st}) \prod_{i=r+1}^{n} L(f, s+\hat{k}-i) .
$$

A persistent theme in the articles of Kurokawa and Miyawaki is the difficulty of computing Fourier coefficients and Euler factors of eigenforms. The Fourier coefficients for our examples were computed in [18] and here we use Krieg's matrix [14] to compute Euler factors from Hecke eigenvalues. In particular, we compute Euler 2-factors for all of the Hecke eigencuspforms in degree four where 
$\operatorname{dim} S_{4}^{k}$ is currently known, namely $k \leq 16$. Two of our examples look suspiciously like unknown lifts because their Euler 2-factors possess rational factors of lower degree and because not all of their Satake parameters are unimodular. However, we cannot identify these factors with Euler factors of eigenforms of lower degree and so leave the matter as lifting puzzles.

The most interesting case is weight 16 with $\operatorname{dim} S_{4}^{16}=7$, see Table 2 . Here there are three pairs of eigenforms with quadratic irrationalities and one rational eigenform. Two eigenforms, $h_{1}$ and $h_{2}$, are Ikeda lifts. Two eigenforms, $h_{5}$ and $h_{6}$, are Miyawaki lifts and we prove this by verifying the nonvanishing of the relevant inner product. Another pair, $h_{3}$ and $h_{4}$, have Euler 2-factors which factor into elliptic factors and a rational quartic factor of unknown origin. Neither do we know what to make of the rational form $h_{7}$, whose Euler 2-factor has no unimodular roots.

We summarize the results of our computations: For an elliptic modular eigenform $f \in S_{1}^{k}$, we may normalize $f$ so that the Fourier expansion $f(\tau)=\sum_{j=1}^{\infty} a(j ; f) q^{j}$ satisfies $a(1 ; f)=1$. The Hecke $L$-function is then defined by

$$
L(f, s)=\prod_{p \text { prime }} Q_{p}\left(f, p^{-s}\right)^{-1} \quad \text { where } Q_{p}(f, X)=1-a(p ; f) X+p^{k-1} X^{2} .
$$

Let $\phi_{28}^{ \pm}(\tau)=q+(-4140 \pm 108 \beta) q^{2}+\cdots$ with $\beta=\sqrt{18209}$ be the two normalized eigenforms of weight 28 . The standard $L$-function of the Ikeda lift $I_{4}\left(\phi_{28}^{ \pm}\right) \in S_{4}^{16}$ is

$L\left(I_{4}\left(\phi_{28}^{ \pm}\right), s, \mathrm{st}\right)=\zeta(s) L\left(\phi_{28}^{ \pm}, s+12\right) L\left(\phi_{28}^{ \pm}, s+13\right) L\left(\phi_{28}^{ \pm}, s+14\right) L\left(\phi_{28}^{ \pm}, s+15\right)$.

The Euler 2-factor is given by

$$
\begin{aligned}
(1-X) Q_{2}\left(\phi_{28}^{ \pm}, 2^{-12} X\right) Q_{2}\left(\phi_{28}^{ \pm}, 2^{-13} X\right) Q_{2}\left(\phi_{28}^{ \pm}, 2^{-14} X\right) Q_{2}\left(\phi_{28}^{ \pm}, 2^{-15} X\right) \\
=(1-X)\left(1-2^{-12}(-4140 \pm 108 \beta) X+2^{3} X^{2}\right) \\
\quad \times\left(1-2^{-13}(-4140 \pm 108 \beta) X+2^{1} X^{2}\right) \\
\quad \times\left(1-2^{-14}(-4140 \pm 108 \beta) X+2^{-1} X^{2}\right) \\
\quad \times\left(1-2^{-15}(-4140 \pm 108 \beta) X+2^{-3} X^{2}\right) .
\end{aligned}
$$

These are exactly the Euler 2-factors computed for $h_{1}$ and $h_{2}$ in Table 2, so that $h_{1}$ is the Ikeda lift of $\phi_{28}^{+}$to degree four and $h_{2}$ is the lift of $\phi_{28}^{-}$.

To identify $h_{5}$ and $h_{6}$ as Miyawaki lifts, let $\phi_{26}(\tau)=q-48 q^{2}+\cdots$ be the normalized eigenform of weight 26 and $\phi_{30}^{ \pm}(\tau)=q+(4320 \pm 96 \gamma) q^{2}+\cdots$ with $\gamma=\sqrt{51349}$ be the two normalized eigenforms of weight 30 . The two SaitoKurokawa lifts $I_{2}\left(\phi_{30}^{ \pm}\right)$span $S_{2}^{16}$. In Section 5 we prove that $M_{4}\left(\phi_{26}, I_{2}\left(\phi_{30}^{ \pm}\right)\right)$is nontrivial, so that it is an eigenform with standard $L$-function

$$
\begin{gathered}
L\left(M_{4}\left(\phi_{26}, I_{2}\left(\phi_{30}^{ \pm}\right)\right), s, \mathrm{st}\right)=L\left(I_{2}\left(\phi_{30}^{ \pm}\right), s, \mathrm{st}\right) L\left(\phi_{26}, s+12\right) L\left(\phi_{26}, s+13\right) \\
=\zeta(s) L\left(\phi_{30}^{ \pm}, s+14\right) L\left(\phi_{30}^{ \pm}, s+15\right) L\left(\phi_{26}, s+12\right) L\left(\phi_{26}, s+13\right) .
\end{gathered}
$$


The Euler 2-factor is given by

$$
\begin{aligned}
& (1-X)\left(1-2^{-14}(4320 \pm 96 \gamma) X+2^{1} X^{2}\right)\left(1-2^{-15}(4320 \pm 96 \gamma) X+2^{-1} X^{2}\right) \\
& \quad \times\left(1-2^{-12}(-48) X+2^{1} X^{2}\right)\left(1-2^{-13}(-48) X+2^{-1} X^{2}\right) .
\end{aligned}
$$

From Table 2 we see that $M_{4}\left(\phi_{26}, I_{2}\left(\phi_{30}^{+}\right)\right)$gives $h_{5}$ and $M_{4}\left(\phi_{26}, I_{2}\left(\phi_{30}^{-}\right)\right)$gives $h_{6}$.

Consider the pair $h_{3}$ and $h_{4}$. From Table 2, the Euler 2-factor of $h_{3}$ is given by

$$
\begin{aligned}
Q_{2}\left(h_{3}, X, \text { st }\right)=(1 & -X) Q_{2}\left(\phi_{28}^{+}, 2^{-14} X\right) Q_{2}\left(\phi_{28}^{+}, 2^{-15} X\right) \\
& \times\left(1+\frac{9}{512} X+\frac{1601}{2048} X^{2}+\frac{9}{512} X^{3}+X^{4}\right) .
\end{aligned}
$$

Thus, $h_{3}$ looks like a lift but we do not know the origin of the quartic factor. It is the only factor in Table 2 with unimodular roots, namely $\eta \pm i \sqrt{1-\eta^{2}}$ for $\eta=$ $(9 \pm \sqrt{1277521}) / 2048$. Both quadratic factors, $Q_{2}\left(\phi_{28}^{+}, 2^{-14} X\right)$ and $Q_{2}\left(\phi_{28}^{+}, 2^{-15} X\right)$, are shared with the Euler 2-factor of $h_{1}$ and the Fourier coefficients of $h_{1}$ and $h_{3}$ are both algebraic integers in $\mathbb{Q}(\sqrt{18209})$. We also see that $h_{1}$ and $h_{3}$ are congruence neighbors in the following sense: their Hecke eigenvalues at $p=2$ are all congruent modulo certain primes in the ring of integers of $\mathbb{Q}(\sqrt{18209})$; namely at three, at a prime above five and at a prime above seven.

Finally, consider the Euler 2-factor of the rational form $h_{7}$. If we introduce a pretend Euler factor with an integer parameter $\ell$,

$$
\mathcal{Q}^{ \pm}(X)=1-2^{\ell}(165 \pm 3 \sqrt{764242}) X+2^{21+2 \ell} X^{2},
$$

then we have the following factorization for any choice of $\ell$ :

$$
Q_{2}\left(h_{7}, X \text {, st }\right)=(1-X) \mathcal{Q}^{+}\left(2^{-10-\ell} X\right) \mathcal{Q}^{+}\left(2^{-11-\ell} X\right) \mathcal{Q}^{-}\left(2^{-10-\ell} X\right) \mathcal{Q}^{-}\left(2^{-11-\ell} X\right) \text {. }
$$

These look like Euler factors of weight $22+2 \ell$ with the $s$ variable translated by $10+\ell$ and $11+\ell$. However, none of the level-one elliptic Euler 2-factors from weights $k=24,28,30,32,34$ or 38 have coefficients that generate the quadratic field $\mathbb{Q}(\sqrt{764242})$.

\section{Notation and background}

In this section we give basic definitions and recall theorems of Andrianov and Satake. We define Siegel modular forms, the Hecke algebra, the spherical map and the Satake parameters. For a commutative ring $R$ let $M_{m \times n}(R)$ denote the $R$-module of $m$-by- $n$ matrices with coefficients in $R$. For $x \in M_{m \times n}(R)$ let $x^{\prime} \in M_{n \times m}(R)$ denote the transpose. Let $V_{n}(R)=\left\{x \in M_{n \times n}(R) \mid x^{\prime}=x\right\}$ be the symmetric $n$-by- $n$ matrices over $R ; V_{n}(\mathbb{R})$ is a euclidean vector space under the inner product $\langle x, y\rangle=\operatorname{tr}(x y)$. For $R \subseteq \mathbb{R}$, an element $x \in V_{n}(R)$ is called positive definite, written $x>0$, when $v^{\prime} x v>0$ for all $v \in \mathbb{R}^{n} \backslash\{0\}$; we denote the set of these by $\mathcal{P}_{n}(R)$. The half-integral matrices are $\mathcal{X}_{n}=\left\{T \in \mathcal{P}_{n}(\mathbb{Q}) \mid \forall v \in \mathbb{Z}^{n}, v^{\prime} T v \in \mathbb{Z}\right\}$.

Let $\mathrm{GL}_{n}(R)=\left\{x \in M_{n \times n}(R) \mid \operatorname{det}(x)\right.$ is a unit in $\left.R\right\}$ be the general linear group and $\mathrm{SL}_{n}(R)=\left\{x \in \mathrm{GL}_{n}(R) \mid \operatorname{det}(x)=1\right\}$ the special linear group. For $x \in \mathrm{GL}_{n}(R)$ let 
$x^{*}$ denote the inverse transpose. Let $I_{n} \in \mathrm{GL}_{n}(R)$ be the identity matrix and set $J_{n}=$ $\left(\begin{array}{cc}0 & I_{n} \\ -I_{n} & 0\end{array}\right) \in \mathrm{SL}_{2 n}(R)$. The symplectic group is defined by $\operatorname{Sp}_{n}(R)=\left\{x \in \mathrm{GL}_{2 n}(R)\right.$ $\left.\mid x^{\prime} J_{n} x=J_{n}\right\}$. We write $\Gamma_{n}=\operatorname{Sp}_{n}(\mathbb{Z})$ and for $R \subseteq \mathbb{R}$ define the group of positive $R$-similitudes by $\operatorname{GSp}_{n}^{+}(R)=\left\{x \in M_{2 n \times 2 n}(R) \mid \exists \mu \in R^{+}: g^{\prime} J_{n} g=\mu J_{n}\right\}$. Each $\gamma$ $\in \mathrm{GSp}_{n}^{+}(R)$ has a unique $\mu=\mu(\gamma)=\operatorname{det}(\gamma)^{1 / n}$.

Define the Siegel upper half space $\mathbb{H}_{n}=\left\{\Omega \in V_{n}(\mathbb{C}): \Im \Omega \in \mathcal{P}_{n}(\mathbb{R})\right\}$. The group $\mathrm{GSp}_{n}^{+}(\mathbb{R})$ acts on $\mathbb{H}_{n}$ as $M\langle\Omega\rangle=(A \Omega+B)(C \Omega+D)^{-1}$ for $M=\left(\begin{array}{ll}A & B \\ C & D\end{array}\right)$. For any function $f: \mathbb{H}_{n} \rightarrow \mathbb{C}$ and any $k \in \mathbb{Z}$ we follow Andrianov and let $\langle n\rangle=n(n+1) / 2$ and define the group action for $M \in \mathrm{GSp}_{n}^{+}(R)$ by

$$
\left(\left.f\right|_{k} M\right)(\Omega)=\mu(M)^{k n-\langle n\rangle} \operatorname{det}(C \Omega+D)^{-k} f(M\langle\Omega\rangle) .
$$

The complex vector space of Siegel modular forms of degree $n$ and weight $k$ is denoted by $M_{n}^{k}$ and is defined as the set of holomorphic $f: \mathbb{H}_{n} \rightarrow \mathbb{C}$ such that $\left.f\right|_{k} M=f$ for all $M \in \Gamma_{n}$ and such that for all $Y_{0} \in \mathcal{P}_{n}(\mathbb{R}), f$ is bounded on $\left\{\Omega \in \mathbb{H}_{n} \mid \Im \Omega>Y_{0}\right\}$. For $f \in M_{n}^{k}$ the Siegel $\Phi$-map is defined by $(\Phi f)(\Omega)=\lim _{\lambda \rightarrow+\infty} f\left(\left(\begin{array}{cc}i \lambda & 0 \\ 0 & \Omega\end{array}\right)\right)$ and the space of cusp forms is defined by $S_{n}^{k}=\left\{f \in M_{n}^{k} \mid \Phi f=0\right\}$. Let $e(z)=e^{2 \pi i z}$. By the Koecher principle, an $f \in S_{n}^{k}$ has a Fourier expansion

$$
f(\Omega)=\sum_{T \in \mathcal{X}_{n}} a(T ; f) e(\langle T, \Omega\rangle),
$$

where the $a(T ; f)$ satisfy $a\left(U^{\prime} T U ; f\right)=\operatorname{det}(U)^{k} a(T ; f)$ for all $U \in \mathrm{GL}_{n}(\mathbb{Z})$. The Petersson inner product $\langle f, g\rangle=\int_{F_{n}} \operatorname{det}(Y)^{k-n-1} f(Z) \overline{g(Z)} d Z$ makes $S_{n}^{k}$ into an inner product space. Here the integral is over a fundamental domain $F_{n}$ for the action of $\Gamma_{n}$ on $\mathbb{H}_{n}$ and $Z=X+i Y \in \mathbb{H}_{n}$ and $d Z=\bigwedge_{1 \leq i \leq j \leq n} d X_{i j} \wedge d Y_{i j}$.

We now introduce various Hecke rings. For a group $\Gamma$ contained in a semigroup $S$, the pair $(\Gamma, S)$ is called a Hecke pair if, for all $s \in S, \Gamma \backslash \Gamma s \Gamma$ is finite. The free $\mathbb{Q}$ module on the left cosets $\Gamma s$ with $s \in S$ is denoted by $L(\Gamma, S)$. A right action of $\Gamma$ on $L(\Gamma, S)$ is given by $\Gamma s \mapsto \Gamma s \gamma$ for $\gamma \in \Gamma$ and the $\Gamma$-invariant subspace is denoted by $H(\Gamma, S)$. If we identify a double coset $\Gamma s \Gamma=\bigsqcup_{i} \Gamma s_{i}$ with $\sum_{i} \Gamma s_{i} \in L(\Gamma, S)$, then $H(\Gamma, S)$ is generated by double cosets. The binary operation on $H(\Gamma, S) \times L(\Gamma, S)$ given by $\left(\sum a_{i} \Gamma s_{i}\right)(\Gamma s)=\sum a_{i} \Gamma s_{i} s$ is well defined and restricts to make $H(\Gamma, S)$ an associative ring. We define

$$
\begin{aligned}
\mathcal{S}_{n} & =\mathrm{GSp}_{n}^{+}(\mathbb{Q}) ; & \mathcal{H}_{n} & =H\left(\Gamma_{n}, \mathcal{S}_{n}\right)=\text { the (global) Hecke algebra }, \\
\overline{\mathcal{S}}_{n} & =\mathcal{S}_{n} \cap M_{2 n \times 2 n}(\mathbb{Z}) ; & \overline{\mathcal{H}}_{n} & =H\left(\Gamma_{n}, \overline{\mathcal{S}}_{n}\right)=\text { the integral Hecke algebra, } \\
\mathcal{S}_{n, p} & =\mathcal{S}_{n} \cap \mathrm{GL} \mathrm{L}_{2 n}\left(\mathbb{Z}\left[\frac{1}{p}\right]\right) ; & \mathcal{H}_{n, p} & =H\left(\Gamma_{n}, \mathcal{S}_{n, p}\right)=\text { the } p \text {-part of } \mathcal{H}_{n}, \\
\overline{\mathcal{S}}_{n, p} & =\mathcal{S}_{n, p} \cap M_{2 n \times 2 n}(\mathbb{Z}) ; & \overline{\mathcal{H}}_{n, p} & =H\left(\Gamma_{n}, \overline{\mathcal{S}}_{n, p}\right)=\text { the } p \text {-part of } \overline{\mathcal{H}}_{n} .
\end{aligned}
$$

The Hecke algebra is a commutative ring and acts as a ring of endomorphisms on each $M_{n}^{k}$ via

$$
f\left|\sum_{k} a_{i} \Gamma_{n} s_{i}=\sum_{i} a_{i} f\right|_{k} s_{i},
$$


an action that stabilizes $S_{n}^{k}$. The subalgebra generated by the double cosets $\Gamma_{n} s \Gamma_{n}$ for $s \in \mathrm{GSp}_{n}^{+}(\mathbb{Q})$ with $\mu(s) \in\left(\mathbb{Q}^{\times}\right)^{2}$ is called the even part of the Hecke algebra. Each Hecke operator is self-adjoint with respect to the Petersson inner product and so $M_{n}^{k}$ has a basis of simultaneous eigenforms. We know that $\mathcal{H}_{n}$ is generated by $\cup_{p} \mathcal{H}_{n, p}$ and that $\mathcal{H}_{n, p}$ is generated by $\overline{\mathcal{H}}_{n, p}$ and $\Gamma_{n}\left(p I_{2 n}\right)^{-1}$. The $p$-part of the integral Hecke algebra, $\overline{\mathcal{H}}_{n, p}$, is generated by the double cosets, for $1 \leq i \leq n$ :

$$
T(p)=\Gamma_{n} \operatorname{diag}\left(I_{n} ; p I_{n}\right) \Gamma_{n} \quad \text { and } \quad T_{i}\left(p^{2}\right)=\Gamma_{n} \operatorname{diag}\left(I_{n-i}, p I_{i} ; p^{2} I_{n-i}, p I_{i}\right) \Gamma_{n} .
$$

We also define $T_{0}\left(p^{2}\right)=\Gamma_{n} \operatorname{diag}\left(I_{n} ; p^{2} I_{n}\right) \Gamma_{n}$.

It is no small matter to turn these abstract definitions into a programmable action on the Fourier coefficients. The following formulae in [2] are what we require here. Using the notation in Section 2, for

$$
f(\Omega)=\sum_{T \in \mathcal{X}_{n}} a(T ; f) e(\langle T, \Omega\rangle) \in S_{n}^{k},
$$

the $T$ th coefficient of $f \mid T(p)$ (respectively, $\left.f \mid T_{i}\left(p^{2}\right)\right)$ is given by

$$
a(T ; f \mid T(p))=\sum_{\substack{0 \leq r \leq n \\ U \in G(r, n-r, 0)}} p^{r(r-2 n+2 k-1) / 2} a\left(\frac{1}{p} T[U D(r, n-r, 0)] ; f\right)
$$

and

$$
\begin{aligned}
a\left(T ; f \mid T_{i}\left(p^{2}\right)\right)= & \sum_{\substack{0 \leq r, s \leq n \\
r+s \leq n \\
U \in G(r, s, n-r-s)}} p^{(2 r+s)(k-n+r-1)-r(r-1)} e(T, U) \\
& \times a\left(\frac{1}{p^{2}} T[U D(r, s, n-r-s)] ; f\right) .
\end{aligned}
$$

In the above formulae, use the following notation. Define the exponential sum

$$
e(T, U)=\sum_{\substack{M \text { mod } p \\ \operatorname{rank}_{p}(M)=s-(n-i)}} e\left(\frac{1}{p} \operatorname{tr}\left(T[U] \operatorname{diag}\left(0^{r}, M^{s}, 0^{n-r-s}\right)\right)\right)
$$

where the notation $M^{s}$ indicates that $M \in M_{S \times s}(\mathbb{Q})$ and 0 indicates the zero matrix. The summation is only over symmetric $M$.

The representatives of double cosets are found by enumerating the set $G(r, s, t)$ for $r+s+t=n$, which is the union of the following (extend the notation to let $M^{r, s}$ denote an element of $\left.M_{r \times s}(\mathbb{Q})\right)$ :

$$
\begin{gathered}
\left\{\left(\begin{array}{cc}
x & 1 \\
U & 0
\end{array}\right) \mid U^{(n-1)} \in G(r, s, t-1), x=(p a, 0), a^{1, r} \bmod p\right\} \\
\left\{\left(\begin{array}{cc}
1 & 0 \\
0 & U
\end{array}\right)\left(\begin{array}{ll}
1 & 0 \\
x & I
\end{array}\right) \mid U^{n-1} \in G(r-1, s, t), x=(0, a, b)^{\prime}, a^{1, s} \bmod p, b^{1, t} \bmod p^{2}\right\}
\end{gathered}
$$


and

$$
\begin{gathered}
\left\{\left(\begin{array}{ccc}
0 & 1 & 0 \\
U_{1} & 0 & U_{2}
\end{array}\right)\left(\begin{array}{ccc}
I & 0 & 0 \\
0 & 1 & 0 \\
0 & x & I
\end{array}\right) \mid\left(U_{1}^{n-1, r}, U_{2}^{n-1, n-r-1}\right) \in G(r, s-1, t)\right. \\
\left.x=(0, a)^{\prime}, a^{1, t} \bmod p\right\}
\end{gathered}
$$

where it is understood that $G(r, s, t)=\emptyset$ if any of $r, s$ or $t$ is negative. The only comment we add to these formulae of Breulmann and Kuss is that, in the summation for $e(T, U)$, a zero-dimensional matrix of rank zero must be allowed; that is, we take $e(T, U)=1$ when $i=n$ and $s=0$. These formulae were used to compute the Hecke eigenvalues in Table 1 from the Fourier coefficients at [19].

By a fundamental result of Satake, the $p$-part of the integral Hecke algebra is isomorphic to the invariant ring $\mathbb{Q}\left[x_{0}, \ldots, x_{n}\right]^{W_{n}}$, where $W_{n}$ is the group generated by the permutations of $x_{1}, \ldots, x_{n}$ and by the automorphisms

$$
\tau_{i}\left(x_{0}\right)=x_{0} x_{i}, \quad \tau_{i}\left(x_{i}\right)=x_{i}^{-1}, \quad \tau_{j}\left(x_{i}\right)=x_{i} \quad \text { for } i \neq j .
$$

To define this isomorphism, write left cosets from $\Gamma_{n} \backslash \mathcal{S}_{n, p}$ in the form $\Gamma_{n} M$ with

$$
\mu(M)=p^{\delta} \quad \text { and } \quad M=\left(\begin{array}{cc}
p^{\delta} D^{*} & B \\
0 & D
\end{array}\right) \quad \text { and } \quad D=\left(\begin{array}{cccc}
p^{\delta_{1}} & & & \\
& p^{\delta_{2}} & & * \\
& 0 & \ddots & \\
& & & p^{\delta_{n}}
\end{array}\right)
$$

The terms $p^{\delta}$ and $\mathrm{GL}_{n}(\mathbb{Z}) D$ depend only on the left coset $\Gamma_{n} M$ and the sequence of elementary divisors $p^{\delta_{i}}$ is determined by $\mathrm{GL}_{n}(\mathbb{Z}) D$. Letting

$$
\Omega\left(\Gamma_{n} M\right)=x_{0}^{\delta} \prod_{i=1}^{n}\left(\frac{x_{i}}{p^{i}}\right)^{\delta_{i}}
$$

defines an algebra homomorphism $\Omega: L\left(\Gamma_{n}, \mathcal{S}_{n, p}\right) \rightarrow \mathbb{Q}\left[x_{0}^{ \pm 1}, \ldots, x_{n}^{ \pm 1}\right]$ that induces the Satake isomorphism $\Omega: \overline{\mathcal{H}}_{n, p} \rightarrow \mathbb{Q}\left[x_{0}, \ldots, x_{n}\right]^{W_{n}}$. The map $\Omega$ is a universal object for complex one-dimensional representations of $\mathcal{H}_{n, p}$, see [1, p. 165].

THEOREM 2.1. Let $\lambda \in \operatorname{Hom}_{\mathbb{Q}}\left(\mathcal{H}_{n, p}, \mathbb{C}\right)$ be a nontrivial homomorphism. There is an $\alpha=\left(\alpha_{0}, \alpha_{1}, \ldots, \alpha_{n}\right) \in \mathbb{C}^{n+1}$ such that the following diagram commutes.

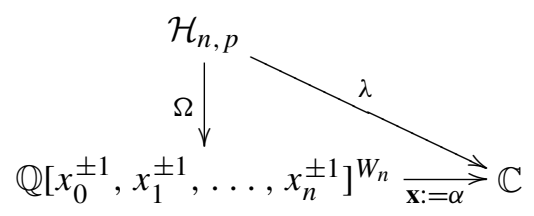

In particular, given an eigenform $F \in S_{n}^{k}$ of the Hecke algebra $\mathcal{H}_{n}$, we may define a homomorphism $\lambda_{F}: \mathcal{H}_{n} \rightarrow \mathbb{C}$ by $\lambda_{F}(T) F=\left.F\right|_{k} T$. For each prime $p$, we have 
TABLE 1. Hecke eigenvalues for all seven eigenforms in $S_{4}^{16}$ as given in [18] where $\beta=\sqrt{18209}$ and $\gamma=\sqrt{51349}$.

\begin{tabular}{|c|c|c|}
\hline \multirow{5}{*}{$h_{1}$} & $T(2)$ & $12960(67989+443 \beta)$ \\
\hline & $T_{0}(4)$ & $276480(2124076963591+2858223465 \beta)$ \\
\hline & $T_{1}(4)$ & $353894400(3255937+7494543 \beta)$ \\
\hline & $T_{2}(4)$ & $-42278584320(-1232945+801 \beta)$ \\
\hline & $T_{3}(4)$ & $515396075520(-523+27 \beta)$ \\
\hline \multirow{5}{*}{$h_{2}$} & $T(2)$ & $-12960(-67989+443 \beta)$ \\
\hline & $T_{0}(4)$ & $-276480(-2124076963591+2858223465 \beta)$ \\
\hline & $T_{1}(4)$ & $-353894400(-3255937+7494543 \beta)$ \\
\hline & $T_{2}(4)$ & $42278584320(1232945+801 \beta)$ \\
\hline & $T_{3}(4)$ & $-515396075520(523+27 \beta)$ \\
\hline \multirow{5}{*}{$h_{3}$} & $T(2)$ & $-230400(1703+9 \beta)$ \\
\hline & $T_{0}(4)$ & $283115520(3556625+644607 \beta)$ \\
\hline & $T_{1}(4)$ & $1698693120(12749123+67821 \beta)$ \\
\hline & $T_{2}(4)$ & $43486543872(183579+533 \beta)$ \\
\hline & $T_{3}(4)$ & $206158430208(221+27 \beta)$ \\
\hline \multirow{5}{*}{$h_{4}$} & $T(2)$ & $230400(-1703+9 \beta)$ \\
\hline & $T_{0}(4)$ & $-283115520(-3556625+644607 \beta)$ \\
\hline & $T_{1}(4)$ & $-1698693120(-12749123+67821 \beta)$ \\
\hline & $T_{2}(4)$ & $-43486543872(-183579+533 \beta)$ \\
\hline & $T_{3}(4)$ & $-206158430208(-221+27 \beta)$ \\
\hline \multirow{5}{*}{$h_{5}$} & $T(2)$ & $1175040(557+\gamma)$ \\
\hline & $T_{0}(4)$ & $70778880(1331260505+3483163 \gamma)$ \\
\hline & $T_{1}(4)$ & $18119393280(2397057+11974 \gamma)$ \\
\hline & $T_{2}(4)$ & $38654705664(317997+529 \gamma)$ \\
\hline & $T_{3}(4)$ & $824633720832(449+3 \gamma)$ \\
\hline \multirow{5}{*}{$h_{6}$} & $T(2)$ & $-1175040(-557+\gamma)$ \\
\hline & $T_{0}(4)$ & $-70778880(-1331260505+3483163 \gamma)$ \\
\hline & $T_{1}(4)$ & $-18119393280(-2397057+11974 \gamma)$ \\
\hline & $T_{2}(4)$ & $-38654705664(-317997+529 \gamma)$ \\
\hline & $T_{3}(4)$ & $-824633720832(-449+3 \gamma)$ \\
\hline \multirow{5}{*}{$h_{7}$} & $T(2)$ & 230400000 \\
\hline & $T_{0}(4)$ & 163381183072174080 \\
\hline & $T_{1}(4)$ & -29115328285900800 \\
\hline & $T_{2}(4)$ & -7821199870525440 \\
\hline & $T_{3}(4)$ & 399947354603520 \\
\hline
\end{tabular}


TABLE 2. Euler 2-factors $Q_{2}\left(h_{i}, X\right.$, st $) /(1-X)$ for the eigenforms $h_{i}$ in Table 1 , where $\beta=\sqrt{18209}$ and $\gamma=\sqrt{51349}$.

\begin{tabular}{ll}
\hline & $2^{-46}\left(-1024+(-1035+27 \beta) x-8192 x^{2}\right)$ \\
& $\left(-2048+(-1035+27 \beta) x-4096 x^{2}\right)$ \\
& $\left(-4096+(-1035+27 \beta) x-2048 x^{2}\right)$ \\
& $\left(-8192+(-1035+27 \beta) x-1024 x^{2}\right)$ \\
\hline & $2^{-46}\left(1024+(1035+27 \beta) x+8192 x^{2}\right)$ \\
& $\left(2048+(1035+27 \beta) x+4096 x^{2}\right)$ \\
& $\left(4096+(1035+27 \beta) x+2048 x^{2}\right)$ \\
& $\left(8192+(1035+27 \beta) x+1024 x^{2}\right)$ \\
\hline & $2^{-34}\left(-2048+(-1035+27 \beta) x-4096 x^{2}\right)$ \\
$h_{3}$ & $\left(-4096+(-1035+27 \beta) x-2048 x^{2}\right)$ \\
& $\left(2048+36 x+1601 x^{2}+36 x^{3}+2048 x^{4}\right)$ \\
& $2^{-34}\left(2048+(1035+27 \beta) x+4096 x^{2}\right)$ \\
$h_{4}$ & $\left(4096+(1035+27 \beta) x+2048 x^{2}\right)$ \\
& $\left(2048+36 x+1601 x^{2}+36 x^{3}+2048 x^{4}\right)$ \\
\hline & $2^{-36}\left(-512+(135+3 \gamma) x-1024 x^{2}\right)$ \\
$h_{5}$ & $\left(-1024+(135+3 \gamma) x-512 x^{2}\right)$ \\
& $\left(512+3 x+256 x^{2}\right)$ \\
& $\left(256+3 x+512 x^{2}\right)$ \\
\hline & $2^{-36}\left(512+(-135+3 \gamma) x+1024 x^{2}\right)$ \\
$h_{6}$ & $\left(1024+(-135+3 \gamma) x+512 x^{2}\right)$ \\
& $\left(512+3 x+256 x^{2}\right)$ \\
& $\left(256+3 x+512 x^{2}\right)$ \\
\hline & $2^{-28}\left(32768-5280 x-20755 x^{2}-2640 x^{3}+8192 x^{4}\right)$ \\
& $\left(8192-2640 x-20755 x^{2}-5280 x^{3}+32768 x^{4}\right)$ \\
\hline &
\end{tabular}

the restriction $\lambda_{F}: \mathcal{H}_{n, p} \rightarrow \mathbb{C}$ and the $\left(\alpha_{0, p}, \ldots, \alpha_{n, p}\right)$ promised in Theorem 2.1 are called the Satake p-parameters of $F$ and satisfy $\left.\Omega(T)\right|_{\mathbf{x}:=\alpha}=\lambda_{F}(T)$ for $T \in \mathcal{H}_{n, p}$. The Satake parameters of $F$ are used in the definition of the $L$-functions attached to $F$. In particular, the standard $L$-function is given by

$$
L(F, s, \mathrm{st})=\prod_{p \text { prime }} Q_{p}\left(\alpha_{p}, p^{-s}\right)^{-1}
$$

where

$$
Q_{p}(F, X, \text { st })=Q_{p}\left(\alpha_{p}, X\right)=(1-X) \prod_{i=1}^{n}\left(1-\alpha_{i, p} X\right)\left(1-\alpha_{i, p}^{-1} X\right) .
$$


The spinor $L$-function is given by

$$
L(F, s, \operatorname{spin})=\prod_{p \text { prime }} Q_{p}\left(F, p^{-s}, \operatorname{spin}\right)^{-1}
$$

where

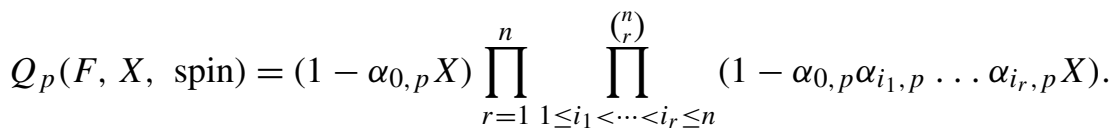

\section{Computing Euler factors}

In recent work the first and third authors have computed bases for the spaces of degree four Hecke eigenforms in weights up to 16. In what follows, we show how to compute the Hecke eigenvalues of these forms and from this deduce their Satake parameters at the prime two. In all cases, the standard $L$-functions factor, suggesting that all of the forms in [18] are lifts. All but three of the computed forms we identify as either Ikeda lifts or Miyawaki lifts, leaving us to wonder whether there are two previously unidentified lifts into genus four.

In what follows, we make use of the following notation. For a polynomial $f \in$ $\mathbb{Q}\left[x_{0}, \ldots, x_{n}\right]$ we set $(\sigma \cdot f)\left(x_{0}, \ldots, x_{n}\right)=f\left(\sigma\left(x_{0}\right), \ldots, \sigma\left(x_{n}\right)\right)$ and set

$$
[f]=\sum_{\sigma \in W_{n} / \operatorname{Stab}(f)} \sigma \cdot f .
$$

Also, if $\mathbf{b}=\left(b_{1}, \ldots, b_{n}\right) \in \mathbb{Z}^{n}$, by $x^{\mathbf{b}}$ we mean the monomial $x_{1}^{b_{1}} \cdots x_{n}^{b_{n}}$. A similar meaning is implied by $\alpha^{\mathbf{b}}$.

Parallel to the generators for $\overline{\mathcal{H}}_{n, p}$, the ring $\mathbb{Q}\left[x_{0}, x_{1}, \ldots, x_{n}\right]^{W_{n}}$ is generated by $x_{0}\left(1+x_{1}\right) \ldots\left(1+x_{n}\right)$ and $\left[x_{0}^{2} x^{(1,1, \ldots, 1)}\right],\left[x_{0}^{2} x^{(2,1, \ldots, 1)}\right], \ldots,\left[x_{0}^{2} x^{(2,2, \ldots, 2,1)}\right]$. Note that, if there are $\ell$ twos in the superscript $(2, \ldots, 2,1, \ldots, 1)$, then

$$
V_{\ell}(\mathbf{x})=\sum_{e_{1}, \ldots e_{n} \in\{1,0,-1\}:\left|e_{1}\right|+\cdots+\left|e_{n}\right|=\ell}^{\left[x^{(2, \ldots, 2,1, \ldots, 1)}\right]=x_{0}^{2} x_{1} \ldots x_{n} V_{\ell}(\mathbf{x}) \text { where }} x_{1}^{e_{1}} x_{2}^{e_{2}} \ldots x_{n}^{e_{n}} .
$$

We note that $V_{\ell}(x)$ is just the $\ell$ th elementary symmetric polynomial in the variables $x_{i}+x_{i}^{-1}$ and we set

$$
V(\mathbf{x})=\left(V_{0}(\mathbf{x}), V_{1}(\mathbf{x}), \ldots, V_{n}(\mathbf{x})\right) .
$$

The generators of $\overline{\mathcal{H}}_{n, p}$ are related to the generators of $\mathbb{Q}\left[x_{0}, x_{1}, \ldots, x_{n}\right]^{W_{n}}$ in the following Theorem [6], see [21] for a more general statement.

THEOREM 3.1. Let $n$ be a positive integer and $p$ a prime. Then

$$
\Omega(T(p))=x_{0}\left(1+x_{1}\right) \ldots\left(1+x_{n}\right) .
$$


Also, there exists an upper triangular matrix $K_{n}\left(p^{2}\right) \in M_{(n+1) \times(n+1)}(\mathbb{Z}[1 / p])$ with positive coefficients on and above the diagonal such that

$$
\begin{aligned}
& \Omega\left(T_{n}\left(p^{2}\right), \ldots, T_{0}\left(p^{2}\right)\right) \\
& \quad=\left(\left[x_{0}^{2} x^{(1,1, \ldots, 1)}\right],\left[x_{0}^{2} x^{(2,1, \ldots, 1)}\right], \ldots,\left[x_{0}^{2} x^{(2,2, \ldots, 2,2)}\right]\right) K_{n}\left(p^{2}\right) .
\end{aligned}
$$

The key computational point is that, for $n \geq 2$, the entries of the matrix $K_{n}\left(p^{2}\right)$ have been given by Krieg [14]. For $n=4$ the Krieg matrix is given by

$$
\left(\begin{array}{ccccc}
\frac{1}{p^{10}} & \frac{p^{4}-1}{p^{10}} & \frac{4 p^{17}-p^{16}-2 p^{14}-p^{12}}{p^{20}} & \frac{\left(p^{2}-1\right)\left(4 p^{17}-p^{16}-2 p^{14}-p^{12}\right)}{p^{20}} & \frac{6 p^{4}-8 p^{3}+2}{p^{4}} \\
0 & \frac{1}{p^{6}} & \frac{p^{3}-1}{p^{6}} & \frac{3 p^{11}-p^{8}\left(1+p+p^{2}\right)}{p^{12}} & \frac{(p-1)\left(3 p^{3}-p^{2}-p-1\right)}{p^{4}} \\
0 & 0 & \frac{1}{p^{3}} & \frac{p^{2}-1}{p^{3}} & \frac{2(p-1)}{p} \\
0 & 0 & 0 & \frac{1}{p} & \frac{p-1}{p} \\
0 & 0 & 0 & 0 & 1
\end{array}\right) .
$$

For general $n$, a Maple program that computes $K_{n}\left(p^{2}\right)$ may be found at [20].

Definition 3.2. For $n \in \mathbb{Z}^{+}$, define $P_{n} \in M_{(n+1) \times(n+1)}(\mathbb{Z})$ by $\left(P_{n}\right)_{i j}=0$ if $i>j$ or $i+j$ is odd and by $\left(P_{n}\right)_{i j}=\left(\begin{array}{c}n+1-i \\ (j-i) / 2\end{array}\right)$ if $i+j$ is even.

For example, in $n=4$,

$$
P_{4}=\left(\begin{array}{lllll}
1 & 0 & 4 & 0 & 6 \\
0 & 1 & 0 & 3 & 0 \\
0 & 0 & 1 & 0 & 2 \\
0 & 0 & 0 & 1 & 0 \\
0 & 0 & 0 & 0 & 1
\end{array}\right)
$$

THEOREM 3.3. Let $n \geq 2$ and $k$ be positive integers and $p$ a prime. Let $F \in M_{n}^{k}$ be a simultaneous eigenform of $\overline{\mathcal{H}}_{n, p}$ with Satake p-parameters $\left(\alpha_{0}, \ldots, \alpha_{n}\right)$. Write the standard Euler p-factor as

$$
Q_{p}(\mathbf{x}, X)=(1-X) \sum_{j=0}^{2 n}(-1)^{j} k_{j}(\mathbf{x}) X^{j} \quad \text { with } k_{j}(\mathbf{x})=k_{2 n-j}(\mathbf{x}) .
$$

If we write

$$
\begin{gathered}
k(\mathbf{x})=\left(k_{0}(\mathbf{x}), k_{1}(\mathbf{x}), \ldots, k_{n}(\mathbf{x})\right), \\
\lambda_{F}\left(p^{2}\right)=\lambda_{F}\left(T_{n}\left(p^{2}\right), T_{n-1}\left(p^{2}\right), \ldots, T_{0}\left(p^{2}\right)\right),
\end{gathered}
$$

then the following relations give $k(\alpha)$ as a linear function of $\lambda_{F}\left(p^{2}\right)$ :

$$
k(\alpha)=V(\alpha) P_{n} \quad \text { and } \quad \lambda_{F}\left(p^{2}\right)=p^{n k-\langle n\rangle} V(\alpha) K_{n}\left(p^{2}\right) .
$$


PROOF. We first note the effect of the slash normalization on the Satake parameters. For any modular form $F \in M_{n}^{k}$,

$$
\left.F\right|_{k} T_{n}\left(p^{2}\right)=\left.F\right|_{k} p \Gamma_{n}=\left(p^{2}\right)^{n k-\langle n\rangle} \operatorname{det}\left(p I_{n}\right)^{-k} F=p^{n k-2\langle n\rangle} F .
$$

Thus, the equality $\lambda_{F}\left(T_{n}\left(p^{2}\right)\right)=p^{n k-2\langle n\rangle}$ is independent of $F$. Since $T_{n}\left(p^{2}\right)=p \Gamma_{n}$ consists of one coset, we may compute $\Omega\left(T_{n}\left(p^{2}\right)\right)=p^{-\langle n\rangle} x_{0}^{2} x_{1} \ldots x_{n}$ directly from the definition. Therefore, the Satake parameters of an eigenform $F$ satisfy $\alpha_{0}^{2} \alpha_{1} \ldots \alpha_{n}=p^{n k-\langle n\rangle}$.

In the defining relation of the Krieg matrix, $K_{n}\left(p^{2}\right)$,

$$
\begin{aligned}
\Omega\left(T_{n}\left(p^{2}\right), \ldots, T_{0}\left(p^{2}\right)\right)= & \left(\left[x_{0}^{2} x^{(1,1, \ldots, 1)}\right],\left[x_{0}^{2} x^{(2,1, \ldots, 1)}\right], \ldots,\left[x_{0}^{2} x^{(2,2, \ldots, 2,2)}\right]\right) \\
& \times K_{n}\left(p^{2}\right)
\end{aligned}
$$

we may substitute the Satake parameters $\mathbf{x}:=\alpha$ to obtain

$$
\lambda_{F}\left(p^{2}\right)=p^{n k-\langle n\rangle} V(\alpha) K_{n}\left(p^{2}\right) .
$$

The other relation, $k(\mathbf{x})=V(\mathbf{x}) P_{n}$, is combinatorial; for example, when $\ell$ is even, the coefficient of $X^{\ell}$ in $\prod_{i=1}^{n}\left(1-\left(x_{i}+x_{i}^{-1}\right) X+X^{2}\right)$ is

$$
V_{\ell}(\mathbf{x})+\left(\begin{array}{c}
n-(\ell-2) \\
1
\end{array}\right) V_{\ell-2}(\mathbf{x})+\left(\begin{array}{c}
n-(\ell-4) \\
2
\end{array}\right) V_{\ell-4}(\mathbf{x})+\cdots .
$$

With these formulae from the Krieg matrix we may compute the Euler 2-factors in Table 2 from the Hecke eigenvalues in Table 1. As a check on computations, one may use the following relation.

THEOREM 3.4. For $n \geq 2$, the following relation holds in $\overline{\mathcal{H}}_{n, p}$ :

$$
\begin{gathered}
T(p)^{2}=T_{0}\left(p^{2}\right)+(1+p) T_{1}\left(p^{2}\right)+(1+p)\left(1+p^{2}\right) T_{2}\left(p^{2}\right)+\cdots \\
+(1+p) \ldots\left(1+p^{n}\right) T_{n}\left(p^{2}\right) .
\end{gathered}
$$

Proof. This is Proposition 5.1 in Hafner-Walling [7], rewritten in Andrianov's notation.

\section{Computing Duke-Imamoḡlu-Ikeda lifts}

Let $n, k$ and $(n+k) / 2$ be even. Let $\phi \in S_{1}^{k}$ be an eigenform. The Ikeda lift of $\phi$ to $I_{n}(\phi) \in S_{n}^{(n+k) / 2}$ is described in [9]. Let $\psi \in S_{1}^{(k+1) / 2}\left(\Gamma_{0}(4)\right)^{+}$be a form corresponding to $\phi$ under the Shimura correspondence. (In this paper we always normalize $\psi$ so that the leading coefficient of its Fourier expansion is one and thereby give meaning to equations such as $I_{4}\left(\phi_{16}\right)=-168 G_{10}$. This somewhat arbitrary choice aids the reproduction of our results and the debugging of code.) 
For a fixed $T \in \mathcal{X}_{n}$, we now describe how to calculate the Fourier coefficient $a\left(T ; I_{n}(\phi)\right)$. For each prime $p$ that divides $\operatorname{det}(2 T)$, along with the prime two, calculate the genus symbol $G_{p}$ of $T$, see [3]. Using the recursion worked out by Katsurada [10], the polynomial $F_{p}$ corresponding to this genus symbol is computed. We refer the reader to [9] or [10] for the definition of the $F_{p}$ polynomial. We thank O. King for giving us his LISP program that computes $F_{p}$ when the genus symbol $G_{p}$ is given as input, see [13].

Use the rational function

$$
\widetilde{F}_{p}(x)=x^{-\left(\operatorname{deg} F_{p}\right) / 2} F_{p}\left(p^{-(n+1) / 2} x\right)
$$

with the property that $\widetilde{F}_{p}\left(x^{-1}\right)=\widetilde{F}_{p}(x)$. Define

$$
b_{p}=p^{-(k-1) / 2}\left(a(p ; \phi)+\sqrt{a(p ; \phi)^{2}-4 p^{k-1}}\right) / 2
$$

so that we have $\left(1-p^{(k-1) / 2} b_{p} x\right)\left(1-p^{(k-1) / 2} b_{p}^{-1} x\right)=1-a(p ; \phi) x+p^{k-1} x^{2}$. Let

$$
c_{T}=\prod_{\text {primes } p: p \mid \operatorname{det}(2 T) \text { or } p=2} \widetilde{F}_{p}\left(b_{p}\right) .
$$

Define a function $\delta: \mathbb{Z} \backslash\{0\} \rightarrow \mathbb{Z} \backslash\{0\}$ by

$$
\delta(y)= \begin{cases}y & \text { if } y \text { is square free and } y \equiv 1 \bmod 4 \\ 4 y & \text { if } y \text { is square free and } y \neq 1 \bmod 4 \\ \delta(\bar{y}) & \text { where } \bar{y} \text { is square free and } y=\ell^{2} \bar{y} \text { for some } \ell \in \mathbb{Z} .\end{cases}
$$

The Ikeda Fourier coefficient at $T$ is

$$
a\left(T ; I_{n}(\phi)\right)=a\left(\left|\delta\left((-1)^{n / 2} \operatorname{det}(2 T)\right)\right| ; \psi\right)\left(\frac{\operatorname{det}(2 T)}{\left|\delta\left((-1)^{n / 2} \operatorname{det}(2 T)\right)\right|}\right)^{(k-1) / 4} c_{T}
$$

\section{Computing Miyawaki-Ikeda lifts}

The Witt maps are given by restriction to the reducible locus, see [22].

PROposition 5.1. For $i+j=n$ there are homomorphisms $\psi_{i j}^{*}: S_{n}^{k} \rightarrow S_{i}^{k} \otimes S_{j}^{k}$ for $\psi_{i j}: \mathbb{H}_{i} \times \mathbb{H}_{j} \rightarrow \mathbb{H}_{n}$ defined by $\psi_{i j}\left(\Omega_{1}, \Omega_{2}\right)=\left(\begin{array}{cc}\Omega_{1} & 0 \\ 0 & \Omega_{2}\end{array}\right)$. Furthermore, if $n=2 i$ we have $\psi_{i i}^{*}: S_{n}^{k} \rightarrow \operatorname{Sym}\left(S_{i}^{k} \otimes S_{i}^{k}\right)$.

The following is essentially due to Ozeki, see [17].

Proposition 5.2. For all $f \in S_{n}^{k}$,

$$
a\left(T_{1} \otimes T_{2} ; \psi_{i j}^{*} f\right)=\sum_{T \in \mathcal{X}_{n}: \pi_{i \times i}^{\text {upper }}(T)=T_{1}, \pi_{j \times j}^{\text {lower }}(T)=T_{2}} a(T ; f) .
$$


The Fourier coefficients of Witt images are typically calculated by the enumeration of $T$ with fixed $T_{1}$ and $T_{2}$; see [18, p. 210] for a specific example.

In the next proposition we use the generators of the ring $M_{2}=\mathbb{C}\left[E_{4}, E_{6}, \chi_{10}, \psi_{12}\right]$ given by Igusa. We refer to [18] for the definition of the various eigenforms $f_{i}$ and for the normalization of Fourier coefficients as well. We let root lattices $A_{4}, D_{4}$, and so on, stand for any Gram matrix of the corresponding lattice, see [3].

Proposition 5.3. In $S_{4}^{14} \otimes S_{2}^{14}$ we have

$$
\psi_{42}^{*} I_{6}\left(\phi_{22}\right)=1848 f_{9} \otimes \chi_{10} E_{4}
$$

Here $f_{9}$ is the element of a Hecke eigenbasis that is not an Ikeda lift, see [18].

PROOF. The space $S_{2}^{14}$ is one dimensional, spanned by $\chi_{10} E_{4}$ and determined by the single Fourier coefficient $a\left(\frac{1}{2} A_{2}\right)$. The space $S_{4}^{14}$ is three dimensional, spanned by $f_{9}$ and two Ikeda lifts $I_{4}\left(\phi_{24}^{ \pm}\right)$and determined by the three Fourier coefficients $a\left(\frac{1}{2} D_{4}\right)$, $a\left(\frac{1}{2} A_{4}\right)$ and $a\left(\frac{1}{2}\left(A_{1} \oplus A_{3}\right)\right)$, see [18, p. 214]. Hence, the element $\psi_{42}^{*} I_{6}\left(\phi_{22}\right)$ of $S_{4}^{14} \otimes$ $S_{2}^{14}$ is determined by the first three of the following four Fourier coefficients. The fourth coefficient is redundant but serves as a check. Computing as in Proposition 5.2 we have

$$
\begin{aligned}
& a\left(\frac{1}{2} D_{4} \otimes\right.\left.\frac{1}{2} A_{2} ; \psi_{42}^{*} I_{6}\left(\phi_{22}\right)\right) \\
&= a\left(\frac{1}{2} D_{4} \oplus \frac{1}{2} A_{2} ; I_{6}\left(\phi_{22}\right)\right)+72 a\left(\frac{1}{2} D_{6} ; I_{6}\left(\phi_{22}\right)\right)+192 a\left(\frac{1}{2} E_{6} ; I_{6}\left(\phi_{22}\right)\right) \\
&= 2784+72(10)+192(1)=3696=1848(2), \\
& a\left(\frac{1}{2} A_{4} \otimes\right.\left.\frac{1}{2} A_{2} ; \psi_{42}^{*} I_{6}\left(\phi_{22}\right)\right) \\
&= a\left(\frac{1}{2} A_{4} \oplus \frac{1}{2} A_{2} ; I_{6}\left(\phi_{22}\right)\right)+120 a\left(\frac{1}{2} D_{6} ; I_{6}\left(\phi_{22}\right)\right)+240 a\left(\frac{1}{2} E_{6} ; I_{6}\left(\phi_{22}\right)\right) \\
& \quad+30 a\left(\frac{1}{2} A_{6} ; I_{6}\left(\phi_{22}\right)\right) \\
&= 8040+120(10)+240(1)+30(-88)=-9240=1848(-5), \\
& a\left(\frac{1}{2}\left(A_{2} \oplus A_{2}\right) \otimes \frac{1}{2} A_{2} ; \psi_{42}^{*} I_{6}\left(\phi_{22}\right)\right) \\
&=a\left(\frac{1}{2}\left(A_{2} \oplus A_{2} \oplus A_{2}\right) ; I_{6}\left(\phi_{22}\right)\right)+432 a\left(\frac{1}{2} D_{6} ; I_{6}\left(\phi_{22}\right)\right)+252 a\left(\frac{1}{2} E_{6} ; I_{6}\left(\phi_{22}\right)\right) \\
& \quad+216 a\left(\frac{1}{2} A_{6} ; I_{6}\left(\phi_{22}\right)\right)+24 a\left(\frac{1}{2} D_{4} \oplus \frac{1}{2} A_{2} ; I_{6}\left(\phi_{22}\right)\right) \\
& \quad+36 a\left(\frac{1}{2} A_{4} \oplus \frac{1}{2} A_{2} ; I_{6}\left(\phi_{22}\right)\right) \\
& \quad+128844+432(10)+252(1)+216(-88) \\
& \quad+24(2784)+36(-8040)=-365904=1848(-198) .
\end{aligned}
$$




$$
\begin{aligned}
a\left(\frac{1}{2}\left(A_{1} \oplus A_{3}\right) \otimes\right. & \left.\frac{1}{2} A_{2} ; \psi_{42}^{*} I_{6}\left(\phi_{22}\right)\right) \\
=a( & \left.\frac{1}{2}\left(A_{1} \oplus A_{3} \oplus A_{2}\right) ; I_{6}\left(\phi_{22}\right)\right)+264 a\left(\frac{1}{2} D_{6} ; I_{6}\left(\phi_{22}\right)\right)+288 a\left(\frac{1}{2} E_{6} ; I_{6}\left(\phi_{22}\right)\right) \\
& +96 a\left(\frac{1}{2} A_{6} ; I_{6}\left(\phi_{22}\right)\right)+90 a\left(\frac{1}{2} D_{5} \oplus \frac{1}{2} A_{1} ; I_{6}\left(\phi_{22}\right)\right) \\
& +24 a\left(\frac{1}{2} A_{5} \oplus \frac{1}{2} A_{1} ; I_{6}\left(\phi_{22}\right)\right)+6 a\left(\frac{1}{2} A_{3} \oplus \frac{1}{2} A_{3} ; I_{6}\left(\phi_{22}\right)\right) \\
= & -54120+264(10)+288(1)+96(-88)+90(-132) \\
& +24(736)+6(17600) \\
= & 51744=1848(28) .
\end{aligned}
$$

The Fourier coefficients $a\left(\cdot ; I_{6}\left(\phi_{22}\right)\right)$ are computed according to Section 4. Since $a\left(\frac{1}{2} D_{4} ; f_{9}\right)=2, a\left(\frac{1}{2} A_{4} ; f_{9}\right)=-5, a\left(\frac{1}{2}\left(A_{2} \oplus A_{2}\right) ; f_{9}\right)=-198$ and $a\left(\frac{1}{2}\left(A_{1} \oplus A_{3}\right) ; f_{9}\right)=28$, see [18, Table 2, p. 218], and $a\left(\frac{1}{2} A_{2} ; \chi_{10} E_{4}\right)=1$ we have the result.

COROLlaRY 5.4. The eigenform $f_{9} \in S_{4}^{14}$ is a Miyawaki lift, namely

$$
M_{4}\left(\phi_{22}, \chi_{10} E_{4}\right)=1848\left\langle\chi_{10} E_{4}, \chi_{10} E_{4}\right\rangle f_{9} .
$$

PROOF. By definition, we have $M_{4}\left(\phi_{22}, \chi_{10} E_{4}\right)=\left\langle\psi_{42}^{*} I_{6}\left(\phi_{22}\right),\left(\chi_{10} E_{4}\right)^{c}\right\rangle$. Since $\chi_{10} E_{4}$ has Fourier coefficients in $\mathbb{Q}$, a totally real field, we have $\left(\chi_{10} E_{4}\right)^{c}=\chi_{10} E_{4}$. By Proposition 5.3, we conclude $\left\langle\psi_{42}^{*} I_{6}\left(\phi_{22}\right),\left(\chi_{10} E_{4}\right)^{c}\right\rangle=\left\langle 1848 f_{9} \otimes \chi_{10} E_{4}, \chi_{10} E_{4}\right\rangle=$ $1848\left\langle\chi_{10} E_{4}, \chi_{10} E_{4}\right\rangle f_{9}$.

A similar but more involved computation gives the following proposition and corollary. Since $S_{2}^{16}$ is two dimensional, we expect to obtain two Miyawaki lifts from the Ikeda lift $I_{6}\left(\phi_{26}\right) \in S_{6}^{16}$.

Proposition 5.5. Let $\omega^{ \pm}=48(1643168 \pm 2141 \gamma) / 200620543$ for $\gamma=\sqrt{51349}$. In $S_{4}^{16} \otimes S_{2}^{16}$ we have

$$
\psi_{42}^{*} I_{6}\left(\phi_{26}\right)=\omega^{-} h_{5} \otimes I_{2}\left(\phi_{30}^{+}\right)+\omega^{+} h_{6} \otimes I_{2}\left(\phi_{30}^{-}\right) .
$$

Corollary 5.6. The eigenforms $h_{5}, h_{6} \in S_{4}^{16}$ are Miyawaki lifts, $M_{4}\left(\phi_{26}\right.$, $\left.I_{2}\left(\phi_{30}^{+}\right)\right)=\omega^{-}\left\langle I_{2}\left(\phi_{30}^{+}\right), I_{2}\left(\phi_{30}^{+}\right)\right\rangle h_{5}$ and $M_{4}\left(\phi_{26}, I_{2}\left(\phi_{30}^{-}\right)\right)=\omega^{+}\left\langle I_{2}\left(\phi_{30}^{-}\right), I_{2}\left(\phi_{30}^{-}\right)\right\rangle h_{6}$.

\section{Final remarks}

Hecke eigenbases of $S_{4}^{k}$ for even $k \leq 16$ were given in [18]. For odd $k \leq 16$, the $S_{4}^{k}$ are trivial, see [4] for some of these results. We survey these standard $L$-functions in degree four. The Schottky form $J_{8}$ spans $S_{4}^{8}$ and $I_{4}\left(\Delta_{12}\right)=-120 J_{8}$ so that

$$
L\left(J_{8}, s, \text { st }\right)=\zeta(s) L\left(\Delta_{12}, s+4\right) L\left(\Delta_{12}, s+5\right) L\left(\Delta_{12}, s+6\right) L\left(\Delta_{12}, s+7\right) .
$$

The form $G_{10}$ spans $S_{4}^{10}$ and $I_{4}\left(\phi_{16}\right)=-168 G_{10}$ so that

$$
L\left(G_{10}, s, \mathrm{st}\right)=\zeta(s) L\left(\phi_{16}, s+6\right) L\left(\phi_{16}, s+7\right) L\left(\phi_{16}, s+8\right) L\left(\phi_{16}, s+9\right) .
$$


The space $S_{4}^{12}=\mathbb{C} f_{5}+\mathbb{C} f_{6}$ has $M_{4}\left(\phi_{18}, I_{2}\left(\phi_{22}\right)\right) /\left\langle I_{2}\left(\phi_{22}\right), I_{2}\left(\phi_{22}\right)\right\rangle=144 f_{6}$ and $I_{4}\left(\phi_{20}\right)=360 f_{5}$. As given by Ikeda [8]:

$$
\begin{gathered}
L\left(f_{5}, s, \mathrm{st}\right)=\zeta(s) L\left(\phi_{20}, s+8\right) L\left(\phi_{20}, s+9\right) L\left(\phi_{20}, s+10\right) L\left(\phi_{20}, s+11\right), \\
L\left(f_{6}, s, \mathrm{st}\right)=\zeta(s) L\left(\phi_{18}, s+8\right) L\left(\phi_{18}, s+9\right) L\left(\phi_{22}, s+10\right) L\left(\phi_{22}, s+11\right) .
\end{gathered}
$$

The space $S_{4}^{14}=\mathbb{C} f_{7}+\mathbb{C} f_{8}+\mathbb{C} f_{9}$ has $M_{4}\left(\phi_{22}, I_{2}\left(\phi_{26}\right)\right) /\left\langle I_{2}\left(\phi_{26}\right), I_{2}\left(\phi_{26}\right)\right\rangle=$ $1848 f_{9}$, and the two Ikeda lifts $I_{4}\left(\phi_{24}^{+}\right)=12 f_{7}$ and $I_{4}\left(\phi_{24}^{-}\right)=12 f_{8}$ :

$$
\begin{aligned}
& L\left(f_{7}, s, \text { st }\right)=\zeta(s) L\left(\phi_{24}^{+}, s+10\right) L\left(\phi_{24}^{+}, s+11\right) L\left(\phi_{24}^{+}, s+12\right) L\left(\phi_{24}^{+}, s+13\right), \\
& L\left(f_{8}, s, \text { st }\right)=\zeta(s) L\left(\phi_{24}^{-}, s+10\right) L\left(\phi_{24}^{-}, s+11\right) L\left(\phi_{24}^{-}, s+12\right) L\left(\phi_{24}^{-}, s+13\right), \\
& L\left(f_{9}, s, \text { st }\right)=\zeta(s) L\left(\phi_{22}, s+10\right) L\left(\phi_{22}, s+11\right) L\left(\phi_{26}, s+12\right) L\left(\phi_{26}, s+13\right) .
\end{aligned}
$$

The $L$-functions for weight 16 , insofar as they are known, were given in the introduction. Recall that the Euler 2-factor of $h_{3}$ is given by

$$
(1-X) Q_{2}\left(\phi_{28}^{+}, 2^{-14} X\right) Q_{2}\left(\phi_{28}^{+}, 2^{-15} X\right)\left(1+\frac{9}{512} X+\frac{1601}{2048} X^{2}+\frac{9}{512} X^{3}+X^{4}\right) .
$$

In the hope of eventually locating this quartic among Euler factors of lower degree, we also give the spinor Euler factor. If

$$
(1-X)\left(1+\frac{9}{512} X+\frac{1601}{2048} X^{2}+\frac{9}{512} X^{3}+X^{4}\right)
$$

were the standard Euler 2-factors of a degree-two eigenform of weight $k$, then the spinor Euler 2-factor would be

$$
\begin{aligned}
1 \pm 2^{k-7} \cdot & 3 \cdot 5^{2} X+2^{2 k-12} \cdot 5 \cdot 7 \cdot 29 X^{2} \pm 2^{3 k-10} \cdot 3 \cdot 5^{2} X^{3}+2^{4 k-6} X^{4} \\
= & \left(1 \pm 2^{k-8} \cdot 3\left(5^{2} \pm \sqrt{641}\right) X+2^{2 k-3} X^{2}\right) \\
& \times\left(1 \pm 2^{k-8} \cdot 3\left(5^{2} \mp \sqrt{641}\right) X+2^{2 k-3} X^{2}\right)
\end{aligned}
$$

Finally, Katsurada has shown that Ikeda lifts have non-Ikeda lifts as congruence neighbors when numerators of certain normalized values of $L$-functions are divisible by large primes. In our examples, we may verify the following: Let $\mathcal{O}$ be the ring of integers of $\mathbb{Q}(\beta)$. The eigenvalues of the Ikeda lift $h_{1}$ and the non-Ikeda lift $h_{3}$ have differences $\lambda_{h_{3}}(T)-\lambda_{h_{1}}(T) \in 3 \mathcal{O} \cap a_{5} \mathcal{O} \cap a_{7} \mathcal{O}$ for $T=T(2), T_{0}(4), T_{1}(4)$, $T_{2}$ (4) and $T_{3}(4)$, where $a_{5}$ of norm 5 and $a_{7}$ of norm 7 are given, respectively, by 3564298180345653559251811701533100061949380892601299566199 $385611273529273539155883991421+26413805826710358011546969364$ $051514900136663884028934590117770380976171799102958519902 \beta$ and 3270594572157720143694411010173434026798776896584037998807223 353400758612485308343317508716 + 24237267926469934357153909329 $439375246810596424836893715023944129121867151461567374501869 \beta$. 


\section{Acknowledgements}

We thank S. Hayashida for helpful discussions. We thank B. Heim for pointing out that Miyawaki lifts are currently only known to be eigenforms for the even part of the Hecke algebra. We thank O. King for giving us a nice LISP program to compute the $F_{p}$ polynomials via Katsurada's recursion formulae. We thank H. Katsurada for bringing his work [11] to our attention.

\section{References}

[1] A. N. Andrianov, Quadratic Forms and Hecke Operators, Grundlehren der mathematischen Wissenschaften, 286 (Springer, Berlin, 1987).

[2] S. Breulmann and M. Kuss, 'On a conjecture of Duke-Imamoḡlu', Proc. Amer. Math. Soc. 128 (2000), 1595-1604.

[3] J. H. Conway and N. J. A. Sloane, Sphere Packings, Lattices and Groups, 3rd edn, Grundlehren der mathematischen Wissenschaften, 290 (Springer, New York, 1999).

[4] W. Duke and Ö. Imamoḡlu, 'Siegel modular forms of small weight', Math. Ann. 310 (1998), 73-82.

[5] M. Eichler and D. Zagier, The Theory of Jacobi Forms, Progress in Mathematics, 55 (Birkhäuser, Basel, 1985).

[6] E. Freitag, Siegelsche Modulfunktionen, Grundlehren der mathematischen Wissenschaften, 254 (Springer, Berlin, 1983), p. $x+341$.

[7] J. L. Hafner and L. H. Walling, 'Explicit action of Hecke operators on Siegel modular forms', J. Number Theory 93 (2002), 34-57.

[8] T. Ikeda, 'Pullback of the lifting of elliptic cusp forms and Miyawaki's conjecture', Duke Math J. 131 (2006), 469-497.

[9] , 'On the lifting of elliptic cusp forms to Siegel cusp forms of degree 2n', Ann. of Math. (2) 154 (2001), 641-681.

[10] H. Katsurada, 'An explicit formula for Siegel series', Amer. J. Math. 121 (1999), 415-452.

[11] H. Katsurada and H. Kawamura, 'On Ikeda's conjecture on the period of the Ikeda lift and its application', in: RIMS Kokyuroku Bessatsu (RIMS Lecture Notes, Extra Volume) (Research Institute for Mathematical Sciences, Kyoto, 2008), pp. 1-22.

[12] - On Ikeda's conjecture on the period of the Ikeda lift, Proc. RIMS Workshop on Construction of Automorphic Forms and their Periods with Applications to appear.

[13] O. D. King, 'A mass formula for unimodular lattices with no roots', Math. Comp. 72 (2003), 839-863.

[14] A. Krieg, 'Das vertauschungsgesetz zwischen Hecke-Operatoren und dem Siegelschen $\phi$-Operator', Arch. Math. (Basel) 46 (1986), 323-329.

[15] N. Kurokawa, 'Examples of eigenvalues of Hecke operators on Siegel cusp forms of degree two', Invent. Math. 49 (1978), 149-165.

[16] I. Miyawaki, 'Numerical examples of Siegel cusp forms of degree 3 and their zeta-functions', Mem. Fac. Sci. Kyushu Univ. Ser. A 46 (1992), 307-339.

[17] M. Ozeki, 'On a property of Siegel theta-series', Math. Ann. 228 (1977), 249-258.

[18] C. Poor and D. S. Yuen, 'Computations of Spaces of Siegel modular cusp forms', J. Math. Soc. Japan 59 (2007), 185-222.

[19] - Authors website, http://math.lfc.edu/ yuen/comp.

[20] - Authors website, http://math.lfc.edu/ yuen/puzzles.

[21] N. C. Ryan and T. R. Shemanske, 'Inverting the satake isomorphism for $S p_{n}$ with applications to Hecke operators', Ramanujan J. 17 (2008), 219-244.

[22] E. Witt, 'Eine Identität zwischen Modulformen zweiten Grades', Abh. Math. Sem. Hansischen Univ. 14 (1941), 323-337. 
CRIS POOR, Department of Mathematics, Fordham University, Bronx, NY 10458-5165, USA

e-mail: poor@fordham.edu

NATHAN C. RYAN, Department of Mathematics, Bucknell University, Lewisburg, PA 17837, USA

e-mail: nathan.ryan@bucknell.edu

DAVID S. YUEN, Department of Mathematics and Computer Science, Lake Forest College, 555 North Sheridan Road, Lake Forest, IL 60045, USA

e-mail: yuen@lakeforest.edu 